Article

\title{
Challenges in a Hybrid Fabrication Process to Generate Metallic Polarization Elements with Sub-Wavelength Dimensions
}

\author{
Stefan Belle *, Babette Goetzendorfer and Ralf Hellmann \\ Applied Laser and Photonics Group, Aschaffenburg University of Applied Sciences, Wuerzburger Str. 45, \\ 63743 Aschaffenburg, Germany; babette.goetzendorfer@th-ab.de (B.G.); ralf.hellmann@th-ab.de (R.H.) \\ * Correspondence: stefan.belle@th-ab.de
}

Received: 6 October 2020; Accepted: 19 November 2020; Published: 22 November 2020

\begin{abstract}
We report on the challenges in a hybrid sub-micrometer fabrication process while using three dimensional femtosecond direct laser writing and electroplating. With this hybrid subtractive and additive fabrication process, it is possible to generate metallic polarization elements with sub-wavelength dimensions of less than $400 \mathrm{~nm}$ in the cladding area. We show approaches for improving the adhesion of freestanding photoresist pillars as well as of the metallic cladding area, and we also demonstrate the avoidance of an inhibition layer and sticking of the freestanding pillars. Three-dimensional direct laser writing in a positive tone photoresist is used as a subtractive process to fabricate free-standing non-metallic photoresist pillars with an area of about $850 \mathrm{~nm} \times 1400 \mathrm{~nm}$, a height of $3000 \mathrm{~nm}$, and a distance between the pillars of less than $400 \mathrm{~nm}$. In a subsequent additive fabrication process, these channels are filled with gold by electrochemical deposition up to a final height of $2200 \mathrm{~nm}$. Finally, the polarization elements are characterized by measuring the degree of polarization in order to show their behavior as quarter- and half-wave plates.
\end{abstract}

Keywords: hybrid fabrication process; three-dimensional direct laser writing; hollow waveguide array; metallic sub-wavelength structures; quarter- and half-wave plate

\section{Introduction}

Laser material processing technologies comprise innumerable subtractive and additive manufacturing approaches. Typical subtractive, material-removing, processes are, e.g., laser modification and removal [1,2], laser ablation [3,4], laser micro-drilling [5,6], laser cutting [7,8], and laser structuring $[9,10]$. Laser-based additive manufacturing encompasses, most commonly, stereolithography [11,12], selective laser melting [13-15], laser sintering [16,17], or laser metal deposition $[18,19]$. In addition to these, predominantly for macroscopic components employed technologies, additive manufacturing has been advanced to a technology that is capable of generating structures in the sub-micrometer range through the use of ultrashort pulse lasers. Multiphoton lithography or also known as three-dimensional direct laser writing (3D DLW) uses a tightly focused laser beam from an ultrashort pulse laser to excite a polymerization process in a photosensitive material by multiphoton absorption. The photosensitive material is usually a negative tone photoresist or a hybrid polymer. A three-dimensional movement of the sample and/or of the beam creates three-dimensional complex structures inside the photosensitive material by a pinpoint writing process [20-24].

In this work, the advantages of three-dimensional direct laser writing are used, but the process itself is inverted. By using a positive tone photoresist instead of a negative tone photoresist, the exposed areas are removed after developing and the additive fabrication process becomes a subtractive 
fabrication process. The advantages of 3D DLW, such as the high lateral resolution or the exposure of a limited volume area, are transmittable to the subtractive process and they are necessary for a successful fabrication. Figure 1 shows a schematic drawing of the three-dimensional direct laser writing in a positive tone photoresist. On the left side of Figure 1, the shape and dimensions of the focal volume are shown. Having a width of about $200 \mathrm{~nm}$ and height of about $800 \mathrm{~nm}$ for the given objective with a numerical aperture of 1.4, the polymerized focal volume (voxel) is not large enough for an exposure of the entire height of the photoresist. Therefore, the photoresist must be exposed several times in different levels. By using a lens with a lower numerical aperture, a complete exposure of the photoresist can be achieved. However, this is accompanied by a reduction in the lateral resolution so that the fabrication of photoresist pillars with sub-micrometer dimensions is not possible and the transmission of the final hollow waveguide array would also be reduced. Despite the advantages mentioned, only a few examples of the combination 3D DLW with positive tone photoresist can be found in the literature $[25,26]$. The combination of the subtractive fabrication process, three-dimensional direct laser writing, with the additive fabrication process, electrochemical metal deposition, to a hybrid manufacturing process enables the fabrication of components with extraordinary functions $[27,28]$.

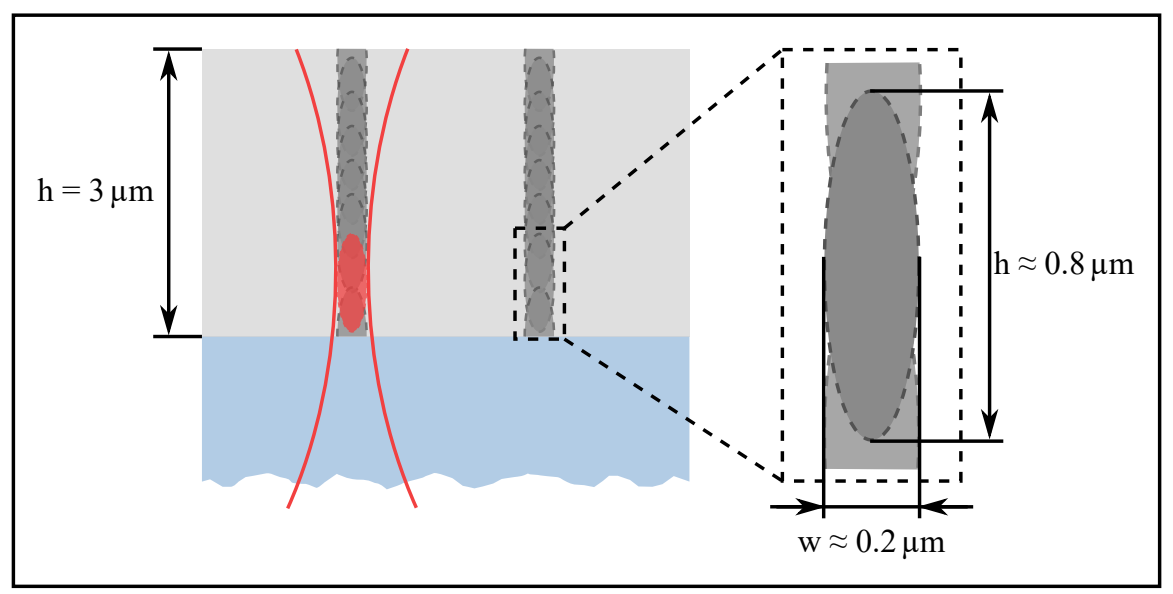

Figure 1. Schematic drawing of a three-dimensional direct laser writing (3D DLW) writing process. The blue area in the figure depicts the substrate, the light gray area the unexposed photoresist, the dark gray areas the exposed photoresist, the red lines approximate the envelope of the laser beam used for exposure, which is strongly focused by an objective with a high numerical aperture and the red area illustrates the focal volume (voxel) in which the polymerization occurs. The right side shows an enlarged illustration of a voxel with the approximate dimensions.

Besides three-dimensional direct laser writing, several methods are conceivable for fabricating such a structure. Metamaterials for the visible wavelength range are fabricated mainly with electron beam lithography (EBL). EBL has a high lateral resolution of a few tens of nanometers, but it has disadvantages in terms of thick resist layers due to electron scattering and the writing speed is low $[29,30]$. For these reasons, EBL is therefore not suitable for the fabrication of the polarization elements presented in this work. Standard UV lithography is also not suitable for fabrication. The minimum line width that is achieved by contact exposure is approximately by a factor of two larger and accordingly reduces the transmission properties of the components [31]. Deep mask-based lithography is a suitable manufacturing process, the achievable resolution is correspondingly high due to the short wavelength of the used source, and, due to the parallel exposure of all structures, is significantly faster than 3D DLW. Disadvantages of this method, however, are the complex mask fabrication for the deep UV exposure and the limited access to a synchrotron radiation source [32]. We decided to use three-dimensional direct laser writing for fabrication due to the advantages of 3D DLW mentioned in the previous paragraph and the arguments listed here. 
The aim of this paper is to show and discuss the challenges of this hybrid subtractive and additive fabrication process by demonstrating a polarization converter as an exemplifying macroscopic component with sub-micrometer function determining the spatial features. In particular, a quarterand a half-waveplate are manufactured, both being based on the concept of hollow waveguides, as theoretically described by Helfert et al. and recently experimentally demonstrated by the authors [33-35].

\section{Basic Theory of Hollow Waveguides}

A hollow waveguide consists of a dielectric medium, in the simplest case ambient air, surrounded by a metallic wall, for example, gold. The lateral dimensions of a hollow waveguide are on a sub-wavelength scale. This dimensions makes it necessary to arrange the hollow waveguides in a field consisting of thousands in order to control the polarization state of a laser beam. Figure 2 illustrates one single waveguide.

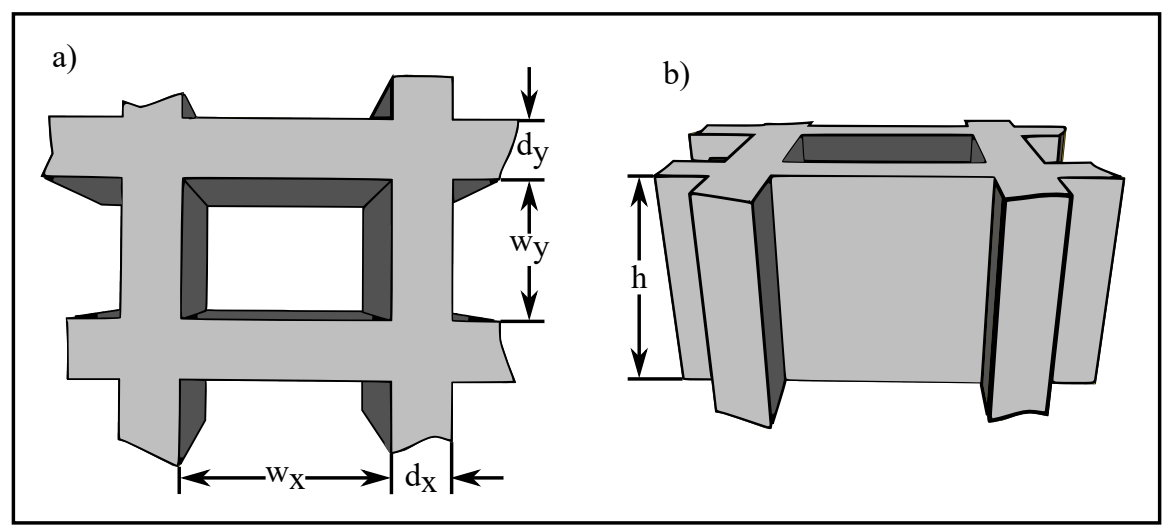

Figure 2. Schematic drawing of a hollow waveguide: (a) shows a top view and (b) shows a tilted side view of a hollow waveguide. The grey-colored structure are the surrounding gold sidewalls. The rectangular spacing in between is the air-filled hollow waveguide.

Using a HWA as a polarization converter for different propagation constants of the vertical and horizontal modes is necessary. This is achieved by using waveguides with a rectangular cross-section. The effective refractive index, which is related to the propagation constant, of these modes can be calculated by [33]

$$
n_{e f f, 10}=\sqrt{1-\frac{\lambda_{0}^{2}}{4 w_{x}^{2}}}
$$

and

$$
n_{e f f, 01}=\sqrt{1-\frac{\lambda_{0}^{2}}{4 w_{y}^{2}}}
$$

with $n_{e f f}$ as the effective refractive index of the horizontal and vertical modes, $\lambda_{0}$ as the free-space wavelength, and $w_{x, y}$ as the width of one single hollow waveguide. The phase delay between the two modes can be determined by

$$
\Delta \Phi=\frac{2 \pi\left(n_{e f f, 10}-n_{e f f, 01}\right) h}{\lambda_{0}}
$$

where $h$ is the height of the HWA. During the fabrication, the height and the width $w_{x}$ of the hollow waveguides is fixed at $h=2200 \mathrm{~nm}$ and $w_{x}=1400 \mathrm{~nm}$. To achieve a phase delay of $270^{\circ}$ (quarter-wave plate) for a free-space wavelength of $1550 \mathrm{~nm}$, a width of $w_{y}=814 \mathrm{~nm}$ is necessary and a width of $w_{y}=884 \mathrm{~nm}$ for a phase delay of $180^{\circ}$ (half-wave plate). 


\section{Fabrication}

The hybrid fabrication process of the polarization element can be divided into two sub-processes. First, the subtractive process for fabricating a negative structure, the non-metallic freestanding photoresist pillars, of the final HWA, and, second, the electrochemical deposition of the metallic sidewalls as an additive process. Figure 3 depicts the complete process chain of the two sub-processes. Please note, the following description of the fabrication process in this chapter is based on the publication of two of the authors in [35] and enriched with further details.

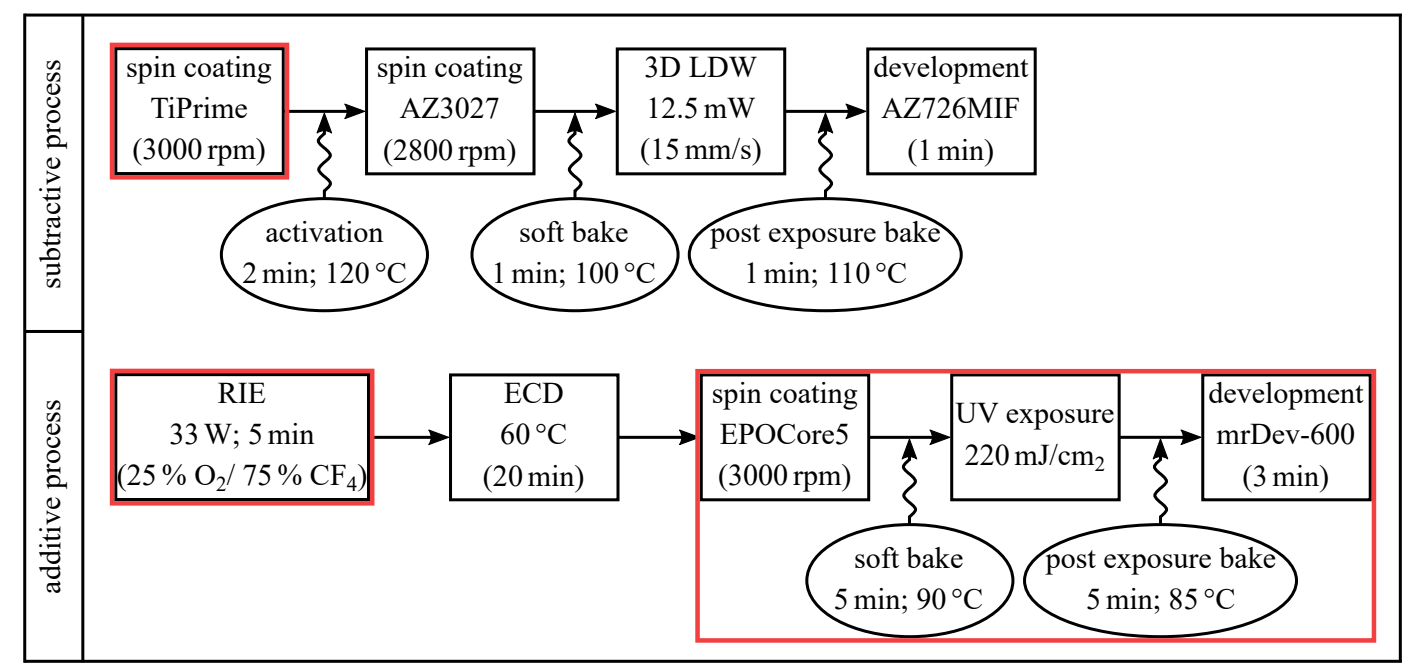

Figure 3. Process chain for the two sub-processes: Top, the fabrication of the negative structure using 3D LDW and a positive tone resist as a subtractive process. Bottom, electrochemical deposition (ECD) of gold as an additive process. The fabrication steps that are marked with a red rectangle are the most challenging process steps during fabrication. These steps are discussed in detail in Section 4, because of their particular importance in the hybrid fabrication process.

For the fabrication of the negative structure, a clean indium tin oxide (ITO) coated glass substrate (Zeiss AG, Oberkochen, Germany) is baked at $140^{\circ}$ for $15 \mathrm{~min}$. for the desorption of residual water molecules deposited on the surface. After this first baking step, an adhesion promotor TiPrime (MicroChemicals GmbH, Ulm, Germany) is spin coated onto the ITO coated glass substrate at $3000 \mathrm{rpm}$ and it is activated in a subsequent, second baking step at $120^{\circ}$ for $2 \mathrm{~min}$. Afterwards, the positive tone photoresist AZ3027 (MicroChemicals $\mathrm{GmbH}$, Germany) is applied by spin coating at $2800 \mathrm{rpm}$. For mechanical and chemical stabilization of the spin coated layer, the sample is baked on a hotplate at $100^{\circ}$ in order to reduce the solvent content of the photoresist. Following, the photoresist is exposed with a 3D DLW-setup Photonic Professional GT (Nanoscribe GmbH, Karlsruhe, Germany). The 3D DLW setup provides $100 \mathrm{fs}$ pulses at $780 \mathrm{~nm}$ with a repetition rate of $80 \mathrm{MHz}$. Here, the writing speed is $15 \mathrm{~mm} / \mathrm{s}$ with an average laser power of $12.5 \mathrm{~mW}$. A total of $36 \times 36$ individual fields with the dimensions of $100 \mu \mathrm{m} \times 100 \mu \mathrm{m}$ for each field are exposed in order to obtain a macroscopic component. The writing time for one field with the writing speed of $15 \mathrm{~mm} / \mathrm{s}$ is $25 \mathrm{~s}$. Immediately after exposure, the resist film is baked at $110^{\circ}$ for $1 \mathrm{~min}$ and then developed in a ready to use developer AZ726MIF (MicroChemicals $\mathrm{GmbH}$, Germany) for $1 \mathrm{~min}$. The developed photoresist layer is thoroughly rinsed with deionized water [35].

After developing the resist film, a disturbing thin inhibition layer is formed on the ITO layer. This thin layer prevents contact between the ITO and the electrolyte and must, therefore, be removed. The additive sub-process begins with the removal of this inhibition layer with an anisotropic reactive ion etching (RIE) step. Anisotropic RIE is performed with a gas mixture of $25 \% \mathrm{O}_{2}$ and $75 \% \mathrm{CF}_{4}$, in a $40 \mathrm{kHz}$ RIE setup Pico (Diener Electronic $\mathrm{GmbH}$, Ebhausen, Germany) with a generator power of $30 \mathrm{~W}$ for $5 \mathrm{~min}$. Further, the ITO coated glass substrate with the freestanding photoresist pillars is built into 
an electrochemical deposition (ECD) chamber and it is placed onto a heating plate at $60^{\circ}$. To achieve a smooth cladding surface and a good mechanical stability, the ECD is performed by alternating pulsed and constant current. The total time for electrochemical deposition is about $20 \mathrm{~min}$., resulting in a gold height of $2200 \mathrm{~nm}$. In order to enhance the adhesion of the gold layer on top of the ITO, an EpoCore5 negative tone photoresist (MicroResist $\mathrm{GmbH}$, Berlin, Germany) is spin coated with a rotational speed of $3000 \mathrm{rpm}$ over the entire structure and softbaked at $90^{\circ}$ for $5 \mathrm{~min}$. Subsequently, the photoresist is exposed by UV-light of a mask aligner EVG620 (EVG GmbH, St. Florian am Inn, Austria) at a dose of $220 \mathrm{~mJ} / \mathrm{cm}^{2}$. After exposure, a hardbake is done at $85^{\circ}$ for $5 \mathrm{~min}$. and the sample is developed in a ready to use developer mr-Dev 600 (MicroResist $\mathrm{GmbH}$, Germany) for $3 \mathrm{~min}$. With this last developing step, a circular area of $2 \mathrm{~mm}$ in diameter is opened and within this circular area also the freestanding pillars are completely removed by the developer. The freestanding photoresist pillars and the final HWA are both shown in Figure 4, right column [35].

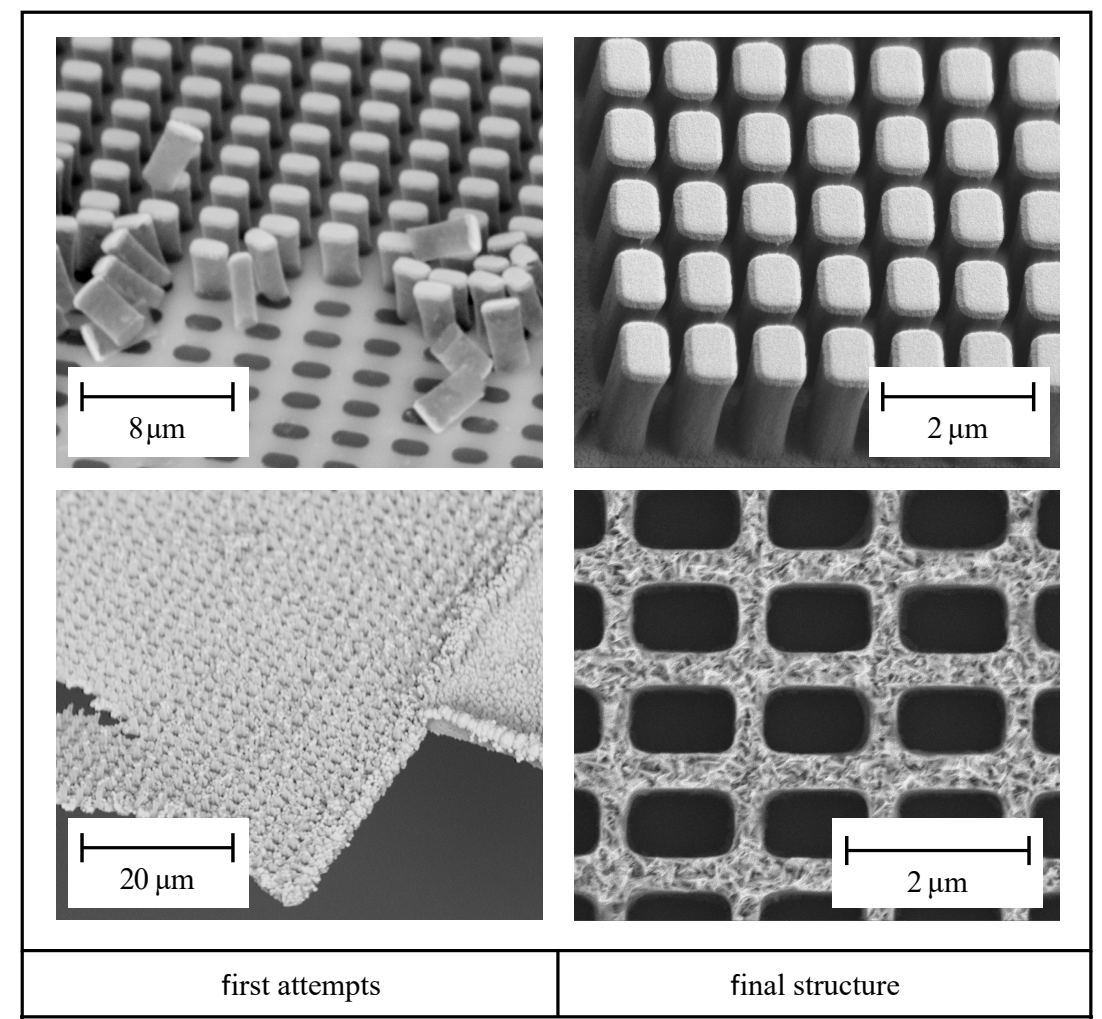

Figure 4. SEM images to compare the challenges from the first attempts (left) to the final fabrication (right). The top images show the freestanding pillars resulting from the subtractive process and the bottom images the final HWA structure.

\section{Fabrication Challenges}

However, the subtractive and additive hybrid process for the fabricaton of metallic optical elements with sub-wavelength dimensions is hallmarked by some challenging process steps, which are marked by red rectangles in the process chain depicted in Figure 3 and that are discussed in detail in the following subsections. In order to illustrate these challenges and, thus, to clarify the importance of the marked process steps for a successful fabrication, Figure 4 contrasts the effects of sub-optimal process conditions (at the beginning of our study on the left) and optimized process settings (final structures on the right). 


\subsection{Weak Adhesion (Part I)}

In order to improve the rather weak adhesion of the freestanding photoresist pillars (Figure 4, top (left)), several treatments of the ITO coated substrate were tested (namely, different combinations of RIE, heat treatment and coating with an adhesion promotor, respectively) and compared to completely untreated substrate properties. Worthwhile to mention, besides improving the adhesion of the photoresist, it is also important to not damage the conductive ITO layer.

Figure 5 summarizes the obtained values for the contact angle $\theta$ (grey), surface roughness $S_{a}$ (red), and sheet resistance $R_{S}$ (blue) for six differently treated substrates $C 1$ to $C 6$, in which $C 1$ represents the untreated substrate.



Figure 5. (a) Experimental characteristics of six differently treated substrates. Grey rhombs = contact angles, red circles = surface roughness and blue squares = difference in sheet resistance relative to untreated substrate. Each column represents one specific treatment. Left to right: column 1 (C1) untreated, C2 baked for $10 \mathrm{~min}, \mathrm{C} 3$ baked for $10 \mathrm{~min}$ and coated with TiPrime, C4 RIE with $\mathrm{O}_{2}$ (100\%, $5 \mathrm{~min}, 30 \mathrm{~W}), \mathrm{C} 5 \mathrm{RIE}$ with $\mathrm{O}_{2} / \mathrm{CF}_{4}\left(25 \% \mathrm{O}_{2} / 75 \% \mathrm{CF}_{4}, 5 \mathrm{~min}, 30 \mathrm{~W}\right), \mathrm{C} 6$ analogue $\mathrm{C} 5$ and coated with TiPrime. (b) Picture of contact angle measurement. Left, contact angle of an untreated substrate (C1). Right, contact angle of a substrate baked for $10 \mathrm{~min}$. and coated with TiPrime (C3).

The contact angle measurements are performed with an optical contact angle measurement setup OCA 25 (DataPhysics Instruments GmbH, Filderstadt, Germany), the surface roughness is measured with an atomic force microscope (AFM) Dimension Icon (Bruker Nano Surfaces Division, Karlsruhe, Germany) and the sheet resistance is determined with a multimeter 34410A (Agilent Technologies Germany GmbH, Waldbronn, Germany) equipped with a substrate holder, built in-house, in order to obtain comparable measurement results. Each coulumn depicts the measured parameter set for a specific substrate treatment.

Apparently, any of the applied surface treatments (C2-C6) reduces the contact angle, which is determined to be around $30^{\circ}$ for the untreated surface (C1). While simply baking the primary substrate results in only a slight decrease of the contact angle $(\mathrm{C} 2)$, all other surface treatments significantly reduce $\theta$. In particular, baking, including coating with TiPrime (C3), reactive ion etching using $\mathrm{O}_{2}$ or $\mathrm{O}_{2} / \mathrm{CF}_{4}(\mathrm{C} 4 \& \mathrm{C} 5)$, or a combination of baking and RIE (C6) clearly reduce the contact angle by up 
to $50 \%$ and, therefore, considerably improve the wetting behavior of the photoresist, advancing the aspired process.

Regarding the surface roughness of the different substrates, simply baking (C2) or baking and coating with TiPrime (C3) shows no distinct effects, as the resulting surface remains rather smooth, i.e., the impact on the surface structure is rather weak and the ITO layer remains intact. In contrast, reactive ion etching steps (C4-C6) lead to an significant increase of the surface roughness from, e.g., $S_{a}=0.162 \mathrm{~nm}$ for $C 2$ up to $0.238 \mathrm{~nm}$ for $C 4$, a trend being in accordance to [36]. This increase in $S_{a}$ is associated with destructive processes within the ITO layer.

For further processing (electroplating), not only the wetting behavior of the photoresist is of high importance, but also the electrochemical behavior of the ITO electrode. Analogue to the application of ITO electrodes in photovoltaic cells, low sheet resistances result in an increase of the electrochemical device efficiency $[37,38]$. As chemical treatments of ITO electrodes influence the electrochemical behavior [36,39], differences in sheet resistances can be seen. Again, simply baking or coating the substrate (C2 and C3) yields no considerable change in sheet resistance (cf. Figure 5 right blue axis and $\Delta R_{S}$ results). In contrast, surface treatment with RIE leads to a clear resistance increase. According to Yu et al. [40], oxygen plasma treatment (C4) leads to the formation of local surface dipoles; therefore, the working function, the electrochemical state, and, consequently, the electrode properties are affected. The use of $\mathrm{O}_{2} / \mathrm{CF}_{4}$ plasma also increases the sheet resistance. This is due to the fact that this kind of plasma treatment allows for creation of ion damage and incorporation of $\mathrm{CF}_{\mathrm{x}}$ into the ITO surface. Therefore, a degradation of electrode performance is also detected [41].

As an interim summary on surface treatment, baking and coating with TiPrime (C3) leads to the most suitable results for further processing. Most importantly, the surface remains intact (low $\mathrm{S}_{\mathrm{a}}$ ), the wetting behavior improves ( $\theta$ decreases), and the electrochemical properties for the following electrochemical Au-deposition remain undisturbed $\left(\Delta \mathrm{R}_{\mathrm{S}}\right.$ remains unaffected on a low level). Furthermore, the process steps are rather simple (baking and coating).

\subsection{Sticking and Inhibition Layer}

As the next steps in the process chain, the substrates are coated with photoresist, exposed by 3D DLW, and developed. However, after developing the photoresist structures, two different effects occur: (i) the photoresist pillars tend to stick together at the top (see Figure $6 \mathrm{~d}$ left) and (ii) in between the pillars an inhibition layer is formed at the ground, which electrically shields the underlying ITO layer.

The sticking of the freestanding photoresist pillars at the top prevents the area between the pillars, denoted here as channels, from being filled with the electrolyte and, in addition, reduces the continuous exchange of used and unused electrolyte. Furthermore, the inhibition layer prevents electrical contact between the electrolyte and the ITO electrode and, thus, counteracts the electrochemical layer deposition. By the use of reactive ion etching, the channels on top are broadened, thus facilitating the electrolyte solution in order to flow into the channels during the electrochemical treatment. In addition, the basic inhibition layer is removed, which prevents contact between electrolyte and ITO electrode.

Figure 6 summarizes and illustrates several effects of four different RIE treatments. Used process gases were $100 \% \mathrm{O}_{2}$ (dotted lines) and a mixture of $25 \% \mathrm{O}_{2}$ and $75 \% \mathrm{CF}_{4}$ (lines), applied for two process powers of $30 \mathrm{~W}$ and $50 \mathrm{~W}$ (red and green), respectively. First, Figure 6a depicts the height of the photoresist pillars versus the duration of the RIE process, revealing a continuous decrease for all RIE steps starting from $2970 \mathrm{~nm}$ for the original pillar after development of the resist. As expected, for both process gases, the height decreases with the applied RIE power, namely from about $20 \%$ in ten minutes for $30 \mathrm{~W}$ to $25 \%$ for $50 \mathrm{~W}$.

Figure $6 \mathrm{~b}$ shows the distance between the individual pillars as a function of RIE process duration for the same RIE process conditions contemplated before in Figure 6a. Because these channels will be filled with gold during the following electroplating step, this distance commensurates with the later gold lattice dimension. Because the thickness of the gold structure, in turn, directly affects the optical transmission properties, this distance should be rather small. The obtained results reveal that the 
distance between individual pillars increases almost linearly with the duration of the etching process, regardless of the employed process gas, yet with a higher slope for higher RIE power. Specifically, for a $30 \mathrm{~W}$ RIE treatment (blue and black) the distance between individual pillars widens from $250 \mathrm{~nm}$ to $450 \mathrm{~nm}$, whereas a $50 \mathrm{~W}$ treatment results in a pillar distance of $550 \mathrm{~nm}$. As an appropriate compromise, an etching time of $5 \mathrm{~min}$ at $30 \mathrm{~W}$ leads to a good geometric basic structure of pillars with $2632 \mathrm{~nm}$ high and a distance of $367 \mathrm{~nm}$.



Figure 6. (a-c) effect of different etching parameters: process gas $\mathrm{O}_{2}$ shown as dotted lines, mixed process gas $25 \% \mathrm{O}_{2} / 75 \% \mathrm{CF}_{4}$ in solid lines. $30 \mathrm{~W}$ power corresponds to the black line (mixed gas) and the blue dotted line (pure $\mathrm{O}_{2}$ ). $50 \mathrm{~W}$ corresponds to the red solid line (mixed gas) and the green dotted line (pure $\mathrm{O}_{2}$ ), repectively. In (a) the pillar height versus etching time is depicted, whereas (b) shows the correlation between etching time and resulting distance in between the pillar structure. The effect of etching on the electrochemical properties of the ITO layer is shown in (c) (for details see Section 4.2). Bottom: (d) REM image of the untreated pillar structure (left) and the final RIE treated structure (right).

On the other hand, the etching process must continue as long as to ensure the complete removal of the inhibition layer in between the pillar structures. Awkwardly, after the complete removal of the inhibition layer, etching will strongly affect the underlying ITO layer and probably destroy it (see Section 4.1). However, AFM measurements for evaluating the quality of the ITO 
layer are performed, as an intact ITO electrode is essential for the following electrochemical gold deposition. Therefore, after RIE treatment, all photoresist pillar structures are removed by using TechniStrip 1316, a common stripper to completely dissolve the photoresist and remove it from the substrate. Consequently, the ITO layer, which was originally underneath the freestanding pillars, should be completely intact. In contrast, areas that are located in the channels in between the original pillar structures should show some effects that result from the preceding etching treatments. However, high precision AFM height measurements reveal no differences in height of the ITO layer at any place, whether the ITO layer was located underneath a pillar structure or in between them. This indicates that no deep physical damage was done by the RIE treatment.

Different current flows throughout the ITO layer can be measured by using a conductive AFM tip and applying a voltage during the measurement. As long as the ITO layer is intact, the resulting current should be the same at any position on the structure. Yet, any damage of the ITO layer should negatively affect the measurable current, which will finally lead to a poorer quality of the electrodeposited gold layer. In order to evaluate the quality of the ITO layer in this respect, the current underneath the pillar structure, as described above dissolved by the stripper, as well as in the channels between the original pillar structure, i.e., areas that were expose to the RIE process, are measured. The difference between these currents $\Delta \mathrm{I}$ is regarded as a qualitative measure of the integrity and quality of the ITO layer with respect to the forthcoming electroplating process step and it is plotted in Figure $6 \mathrm{c}$ as a function of the (RIE) etching time.

The results for $\Delta \mathrm{I}$ in Figure $6 \mathrm{c}$ show no differences up to $2.5 \mathrm{~min}$. etching time $(\Delta \mathrm{I}=0)$, i.e., an intact ITO layer can be assumed at every location on the surface. After $5 \mathrm{~min}$. of etching, however, $\Delta \mathrm{I}$ increases, whic indicates a degradation of the ITO layer and its electrical properties between the original pillar structures. The longer the etching endures, the higher this damage to the ITO layer, which implies poor electrochemical performance during the intended next process step. The results that are shown in Figure $6 \mathrm{c}$ also imply that this effect gets stronger with increasing RIE power, and it is more pronounced for $\mathrm{O}_{2}$ as compared to $\mathrm{O}_{2} / \mathrm{CF}_{4}$ process gases. Altogether, it appears that etching up to $5 \mathrm{~min}$. using $30 \mathrm{~W}$ and $\mathrm{O}_{2} / \mathrm{CF}_{4}$ is a proper compromise to remove the inhibition layer while assuring low abrasion of the ITO layer and, thus, maintaining its electrical function for the electrochemical deposition.

Finally, Figure 6d depicts the SEM images of the photoresist pillars directly after development (left) and after RIE treatment (right). Apparently, directly after developing the freestanding structures almost stick together at the top (left). In addition, the formation of the inhibition layer at the interface with the substrate is also clearly visible. In comparison, the right image clearly shows that the distances between the photoresist pillars are widened and the inhibition layer is removed.

These comprehensive results on the RIE treatment in order to remove the inhibition layer and to eliminate the sticking of adjacent pillars are summarized as follows. The height of the pillars can be well definable reduced by controlling the etching duration in conjunction with the applied power. The particular kind process gas $\left(\mathrm{O}_{2}\right.$ or $\left.\mathrm{O}_{2} / \mathrm{CF}_{4}\right)$ has only minor influence (Figure $\left.6 \mathrm{a}\right)$. The same holds for the widening of the channels between pillar rows (Figure 6b), yet, under any condition, the height reduction is more pronounced as the widening of the channels, because the widening can only start after the shielding pillar tops are removed (cf. Figure $6 \mathrm{~d}$, left). Nonetheless, $5 \mathrm{~min}$. etching using $30 \mathrm{~W}$ appears to sufficiently open access to the channels in between the pillars with acceptable broadening of the channel width. According to Figure $6 c$, these conditions also comply with the necessity of removing the inhibition layer without damaging the ITO, when employing $25 \% \mathrm{O}_{2} / 75 \% \mathrm{CF}_{4}$ process gas.

\subsection{Weak Adhesion (Part II)}

After optimization of the photoresist adhesion (Section 4.1) and after successful preparation for the galvanic gold deposition, the pillars are removed by the second developing step depicted in Figure 3 . Unfortunately, the deposited gold layer is often detached during this step (Figure 4, bottom left). In order to stabilize the gold layered structure, before removing the pillars, a second photoresist layer is 
applied on the complete surface of the sample. This layer is exposed to UV-light irradiation at a circular area of $2 \mathrm{~mm}$ in diameter. After the consecutive developing step, in this circular area, the fixation layer, as well as the photoresist pillar structures, are removed. The outer residual fixation layer acts as a supportive film, so the bare gold structure in the center can be optically characterized. Figure 7 shows the fixation of the metallic hollow waveguide array (HWA) structure on top of the substrate.

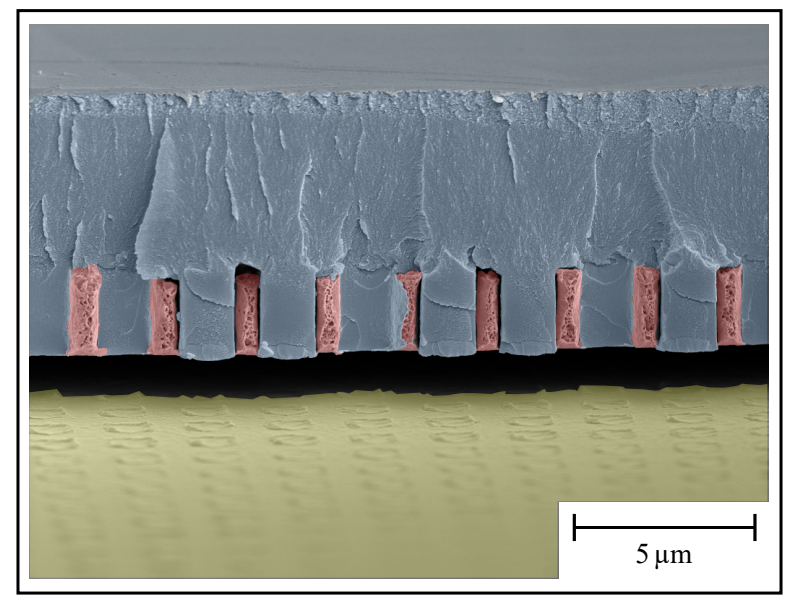

Figure 7. Colorized SEM image of the fixation, side view at an angle of $35^{\circ}$. The red colored areas show the metallic structure, the blue area shows both the freestanding photoresist pillars as well as the fixation layer, and the yellow area shows the substrate. Please note, during preparation for taking this SEM image (by cleaving to allow a side view) the metallic HWA structure as well as the photoresist is detachted from the substrate.

\section{Optical Experiments}

The waveguide dimensions $\left(\mathrm{w}_{\mathrm{x}} \& \mathrm{w}_{\mathrm{y}}\right)$ and the width of the metal side walls $\left(\mathrm{d}_{\mathrm{x}} \& \mathrm{~d}_{\mathrm{y}}\right)$ are measured while using a MAIA3 scanning electron microscope (Tescan GmbH, Dortmund, Germany). In order to determine the waveguide geometry, a total of 25 waveguides are measured at random positions over the entire HWA. For the quarter-wave plate, the dimensions of the hollow waveguides are $\mathrm{w}_{\mathrm{x}}=1403 \pm 7 \mathrm{~nm}$ and $\mathrm{w}_{\mathrm{X}}=815 \pm 3 \mathrm{~nm}$. The half-wave plate has the same dimension $\mathrm{w}_{\mathrm{x}}=1403 \pm 7 \mathrm{~nm}$ in the $\mathrm{x}$-direction as the quarter-wave plate. Only in the y-direction $\mathrm{w}_{\mathrm{y}}=883 \pm 4 \mathrm{~nm}$ both polarization converters differ. For both polarization converters, the dimensions of the metal sidewalls are $d_{x}=396 \pm 6 \mathrm{~nm}$ and $d_{y}=421 \pm 4 \mathrm{~nm}$. The waveguide geometries from the fabricated HWA show excellent agreement with the targeted values of $\mathrm{w}_{\mathrm{x}}=1400 \mathrm{~nm}$ and $\mathrm{w}_{\mathrm{y}}=814 \mathrm{~nm}$ (quarter-wave plate) as well as $\mathrm{w}_{\mathrm{x}}=1400 \mathrm{~nm}$ and $\mathrm{w}_{\mathrm{y}}=884 \mathrm{~nm}$ (half-wave plate). However, the width of the metal sidewalls does not influence the polarization behavior of the polarization converters, only the transmission depends on the width of the sidewalls. While the transmission of the quarter-wave plate is $47.8 \pm 0.13 \%$, the transmission of the half-wave plate amounts to $51.3 \pm 0.18 \%$. These can be assigned by the different waveguide dimensions of $\mathrm{w}_{\mathrm{y}, \lambda / 4}<\mathrm{w}_{\mathrm{y}, \lambda / 2}$. The proportionate higher amount of metal sidewalls on the same square area reduces the transmission due to the smaller waveguide width $\mathrm{w}_{\mathrm{y}}$ of the quarter-wave plate.

The characterization of the fabricated hollow waveguide arrays is done by measuring the minimum and maximum transmitted power and calculate the degree of polarization. The measurement setup consists of a linear polarized laser diode emitting at a wavelength of $\lambda=1550 \mathrm{~nm}$ in constant mode. A beam condensor system is used in order to reduce the beam diameter to $1 \mathrm{~mm}$ and collimate the beam. The hollow waveguide array is mounted in a rotatable holder. After passing through the HWA, the minimum and maximum power is set with an analyzerthat is mounted in a rotatable holder and measured with a power meter. The angle of the hollow waveguide array is stepwise changed from $0^{\circ}$ to $360^{\circ}$ in steps of $5^{\circ}$. For every step, the degree of polarization is calculated by 


$$
D o P=\frac{P_{\max }-P_{\min }}{P_{\max }+P_{\min }}
$$

and the results for both the quarter- and the half-wave plate are shown in Figure 8.

If the degree of polarization assumes a value of 1 , the field after the hollow waveguide array is completely linear polarized. The power transmitted through the analyzer is maximum in the transmission direction and, for a crossed analyzer, the beam is almost completely blocked. The difference between $\left(P_{\max }\right)$ and $\left(P_{\min }\right)$ is maximal and the degree of polarization is calculated according to Equation (4) to 1 . For a value of 0 , the field after the HWA is circularly polarized. A circular polarization has no preferred direction and the transmitted power is equal for every position of the analyzer. According to Equation (4), the degree of polarization is calculated as 0 . For each value between 0 and 1 , the field after the HWA is elliptically polarized.

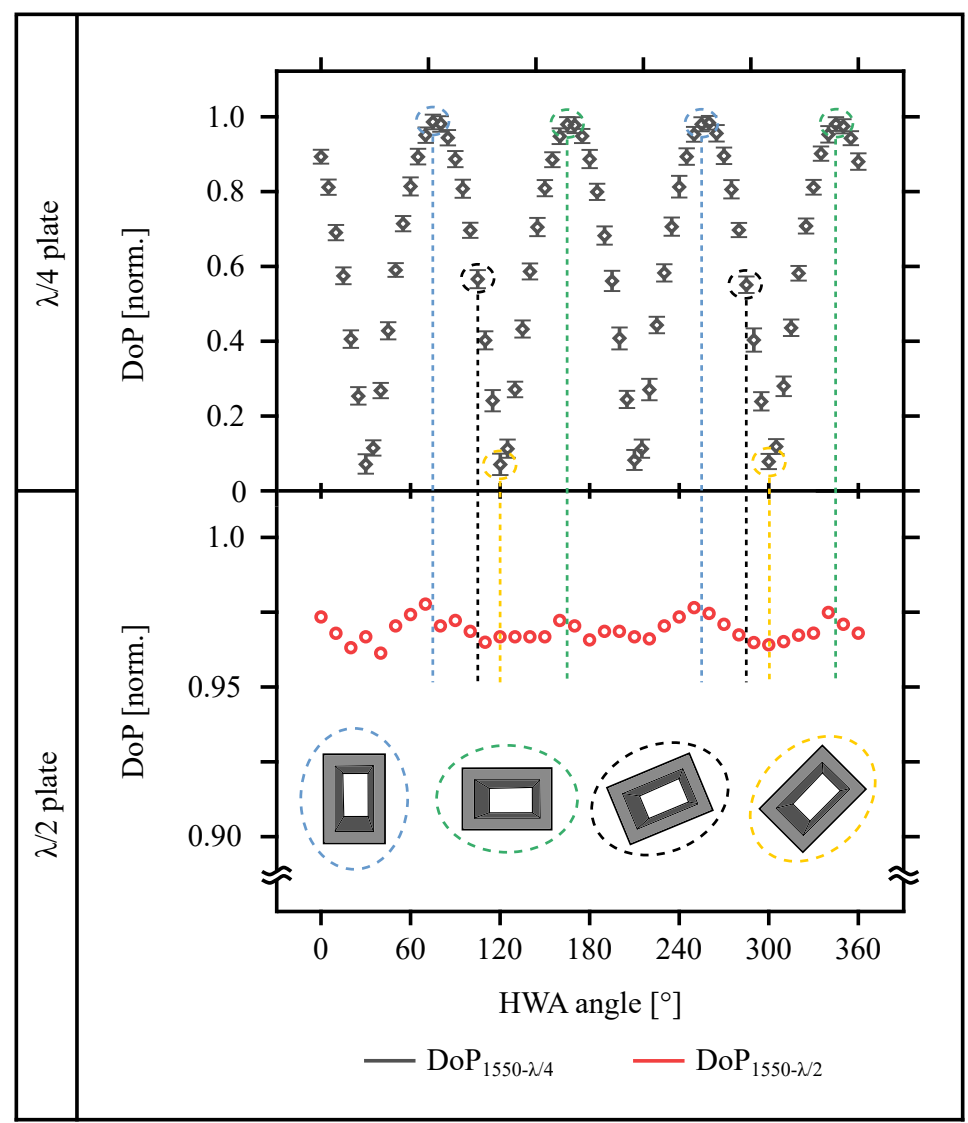

Figure 8. Degree of Polarization against the hollow waveguide array (HWA) angle for a quarter-wave plate (top) and a half-wave plate (bottom). For a clear presentation, the results for the half-wave plate is only plotted in steps of $10^{\circ}$. The schematic drawings of the hollow waveguides in the figure show the orientation of the waveguides for selected positions. These positions are marked in color in the figure. Please note, because of the smaller amount of measurement values for the half-wave plate, no measured value is given for some positions.

The measurements presented in Figure 8 for the quarter-wave plate show four maxima and four minima. The maxima occur when the hollow waveguide array is aligned vertically or horizontally (the blue and green circled hollow waveguides shown in Figure 8, bottom). In the hollow waveguide, only one mode is excited by the linearly polarized input field, no phase delay occur and the linearly polarized input field remains linearly polarized after the hollow waveguide array. The minima occur when the hollow waveguide array is aligned at an angle of $45^{\circ}$ with respect to the horizontal or vertical (the yellow circled hollow waveguide shows two of these four positions). For these four 
positions, the two modes in the hollow waveguide are equally excited and the phase delay is maximal. The maximum phase delay for a quarter-wave plate is $90^{\circ}$, or like shown in this work, $270^{\circ}$. Relating to these values, the linear polarized input field becomes to a circular polarized output field. Every other position of the hollow waveguide array between the positions mentioned above, the field is elliptically polarized. Both of the waveguide modes (horizontal and vertical) are excited with different strengths and depending on the angular position of the HWA, the ellipticity of the polarization state changes. For the half-wave plate (Figure 8, bottom), the degree of polarization remains close to 1 and no distinct extreme values are observable. The maximum phase delay for a half-wave plate is $180^{\circ}$. The linear polarized input field persist linear polarized after the hollow waveguide array, but the field changes their orientation (e.g., a horizontal linear polarized input field becomes a vertical linear output field).

\section{Conclusions}

We have presented a femtosecond three-dimensional direct laser writing based subractive-additive hybrid fabrication process of metallic structures with lateral dimensions of less than $400 \mathrm{~nm}$ and they have exemplified the capabilities of this technological approach by realizing and demonstrating the performance of a fully functional hollow waveguide array based polarization element. In order to facilitate high precision and high quality of such micro-nanostructured components, we comprehensively studied the technological challenges of such sophisticated fabrication process and identified solutions to these. In particular, an appropriate pretreatment of the substrate significantly improves the adhesion of non-metallic freestanding photorosist pillars with rectangular dimensions of $814 \mathrm{~nm} \times 1400 \mathrm{~nm}$ for the quarter-wave plate, $884 \mathrm{~nm} \times 1400 \mathrm{~nm}$ for the half-wave plate, both with a height of $3000 \mathrm{~nm}$, respectively. After developing, a thin inhibition layer forms on the ITO surface and prevents contact between the ITO electrode and the electrolyte, hampering electrochemical deposition. Additionally, the freestanding photoresist pillars tend to stick together at the top because of their high aspect ratio. Both of the challenges are solved with a carefully adjusted reactive ion etching step, removing the occurring inhibition layer and increasing the gap between the pillars. Subsequently, the additive manufactured metallic structure is fixed with a second photoresist layer on top of the substrate. Furthermore, an optical characterization of the two wave plates is performed over their complete angular range and the functionality of a quarter- and half-wave plate that is based on hollow waveguides is shown.

Author Contributions: Conceptualization, S.B. and R.H.; methodology, S.B., B.G. and R.H.; validation, S.B., B.G. and R.H.; formal analysis, S.B.; investigation, S.B. and B.G.; resources, R.H.; data curation, S.B.; writing-original draft preparation, S.B., B.G. and R.H.; writing-review and editing, S.B. and R.H.; visualization, S.B.; supervision, R.H. All authors have read and agreed to the published version of the manuscript.

Funding: This research received no external funding.

Conflicts of Interest: The authors declare no conflict of interest.

\section{References}

1. Gottmann, J.; Hermans, M.; Repiev, N.; Ortmann, J. Selective Laser-Induced Etching of 3D Precision Quartz Glass Components for Microfluidic Applications-Up-Scaling of Complexity and Speed. Micromachines 2017, 8, 110. [CrossRef]

2. Roth, G.L.; Esen, C.; Hellmann, R. Circular microchannels inside bulk polymethylmethacrylate generated by femtosecond laser using slit beam shaping. J. Laser Appl. 2019, 31, 022603. [CrossRef]

3. Mirza, I.; Bulgakova, N.M.; Tomáštík, J.; Michálek, V.; Haderka, O.; Fekete, L.; Mocek, T. Ultrashort pulse laser ablation of dielectrics: Thresholds, mechanisms, role of breakdown. Sci. Rep. 2016, 6, 39133. [CrossRef] [PubMed]

4. Žemaitis, A.; Gečys, P.; Barkauskas, M.; Račiukaitis, G.; Gedvilas, M. Highly-efficient laser ablation of copper by bursts of ultrashort tuneable (fs-ps) pulses. Sci. Rep. 2019, 9, 12280. [CrossRef] [PubMed]

5. Gruner, A.; Naumann, L.; Schille, J.; Loeschner, U. High Pulse Repetition Frequency Micro Hole Drilling of Silicon Using Ultrashort Pulse Laser Radiation. J. Laser Micro Nanoeng. 2019, 14, 190-197. 
6. Shen, N.; Bude, J.D.; Ly, S.; Keller, W.J.; Rubenchik, A.M.; Negres, R.; Guss, G. Enhancement of laser material drilling using high-impulse multi-laser melt ejection. Opt. Express 2019, 27, 19864-19886. [CrossRef] [PubMed]

7. Riveiro, A.; Quintero, F.; Boutinguiza, M.; Val, J.D.; Comesaña, R.; Lusquiños, F.; Pou, J. Laser Cutting: A Review on the Influence of Assist Gas. Materials 2019, 12, 157. [CrossRef]

8. Anghel, C.; Gupta, K.; Jen, T.C. Analysis and optimization of surface quality of stainless steel miniature gears manufactured by CO2 laser cutting. Optik 2020, 203, 164049. [CrossRef]

9. Schwarz, S.; Rung, S.; Esen, C.; Hellmann, R. Fabrication of a high-quality axicon by femtosecond laser ablation and CO2 laser polishing for quasi-Bessel beam generation. Opt. Express 2018, 26, 23287-23294. [CrossRef]

10. Brenner, A.; Osbild, M.; Finger, J. Ultrafast Laser Structuring within a Process Chain - Influence of the Surface Morphology. J. Laser Micro Nanoeng. 2019, 14, 198-205.

11. Melchels, F.P.; Feijen, J.; Grijpma, D.W. A review on stereolithography and its applications in biomedical engineering. Biomaterials 2010, 31, 6121-6130. [CrossRef] [PubMed]

12. Huang, J.; Qin, Q.; Wang, J. A review of stereolithography: Processes and systems. Processes 2020, 8, 1138. [CrossRef]

13. Wüst, P.; Edelmann, A.; Hellmann, R. Areal Surface Roughness Optimization of Maraging Steel Parts Produced by Hybrid Additive Manufacturing. Materials 2020, 13, 418. [CrossRef] [PubMed]

14. Majchrowicz, K.; Pakieła, Z.; Brynk, T.; Romelczyk-Baishya, B.; Płocińska, M.; Kurzynowski, T.; Chlebus, E. Microstructure and mechanical properties of Ti-Re alloys manufactured by selective laser melting. Mater. Sci. Eng. A 2019, 765, 138290. [CrossRef]

15. Zhang, J.; Song, B.; Wei, Q.; Bourell, D.; Shi, Y. A review of selective laser melting of aluminum alloys: Processing, microstructure, property and developing trends. J. Mater. Sci. Technol. 2019, 35, 270-284. [CrossRef]

16. Fina, F.; Goyanes, A.; Gaisford, S.; Basit, A.W. Selective laser sintering (SLS) 3D printing of medicines. Int. J. Pharm. 2017, 529, 285-293. [CrossRef]

17. Sing, S.L.; Yeong, W.Y.; Wiria, F.E.; Tay, B.Y.; Zhao, Z.; Zhao, L.; Tian, Z.; Yang, S. Direct selective laser sintering and melting of ceramics: A review. Rapid Prototyp. J. 2017, 23, 611-623. [CrossRef]

18. Dobbelstein, H.; Gurevich, E.L.; George, E.P.; Ostendorf, A.; Laplanche, G. Laser metal deposition of a refractory TiZrNbHfTa high-entropy alloy. Addit. Manuf. 2018, 24, 386-390. [CrossRef]

19. Carluccio, D.; Bermingham, M.; Kent, D.; Demir, A.G.; Previtali, B.; Dargusch, M.S. Comparative Study of Pure Iron Manufactured by Selective Laser Melting, Laser Metal Deposition, and Casting Processes. Adv. Eng. Mater. 2019, 21, 1900049. [CrossRef]

20. Kawata, S.; Sun, H.B.; Tanaka, T.; Takada, K. Finer features for functional microdevices. Nature 2001, 412, 697-698. [CrossRef]

21. Malinauskas, M.; Farsari, M.; Piskarskas, A.; Juodkazis, S. Ultrafast laser nanostructuring of photopolymers: A decade of advances. Phys. Rep. 2013, 533, 1-31. [CrossRef]

22. Gissibl, T.; Thiele, S.; Herkommer, A.; Giessen, H. Two-photon direct laser writing of ultracompact multi-lens objectives. Nat. Photon. 2016, 10, 554-560. [CrossRef]

23. Zyla, G.; Kovalev, A.; Grafen, M.; Gurevich, E.L.; Esen, C.; Ostendorf, A.; Gorb, S. Generation of bioinspired structural colors via two-photon polymerization. Sci. Rep. 2017, 7, 17622. [CrossRef] [PubMed]

24. Trautmann, A.; Rüth, M.; Lemke, H.D.; Walther, T.; Hellmann, R. Two-photon polymerization based large scaffolds for adhesion and proliferation studies of human primary fibroblasts. Opt. Laser Technol. 2018, 106, 474-480. [CrossRef]

25. Cao, H.Z.; Zheng, M.L.; Dong, X.Z.; Jin, F.; Zhao, Z.S.; Duan, X.M. Two-photon nanolithography of positive photoresist thin film with ultrafast laser direct writing. Appl. Phys. Lett. 2013, 102, 201108. [CrossRef]

26. Ma, Z.C.; Zhang, Y.L.; Han, B.; Chen, Q.D.; Sun, H.B. Femtosecond-Laser Direct Writing of Metallic Micro/ Nanostructures: From Fabrication Strategies to Future Applications. Small Methods 2018, 2, 1700413. [CrossRef]

27. Gansel, J.K.; Thiel, M.; Rill, M.S.; Decker, M.; Bade, K.; Saile, V.; von Freymann, G.; Linden, S.; Wegener, M. Gold helix photonic metamaterial as broadband circular polarizer. Science 2009, 325, 1513-1515. [CrossRef]

28. Wendy Gu, X.; Greer, J.R. Ultra-strong architected Cu meso-lattices. Extrem. Mech. Lett. 2015, 2, 7-14. [CrossRef] 
29. Grigorescu, A.E.; Hagen, C.W. Resists for Sub-20-nm Electron Beam Lithography with a Focus on HSQ: State of the Art. Nanotechnology 2009, 20, 292001. [CrossRef]

30. She, A.; Zhang, S.; Shian, S.; Clark, D.R.; Capasso, F. Large area metalenses: Design, characterization, and mass manufacturing. Opt. Express 2018, 26, 1573-1585. [CrossRef]

31. Ni, H.; Yuan, G.; Sun, L.; Chang, N.; Di, Z.; Chen, R.; Jiang, L.; Chen, H.; Gu, Z.; Zhao, X. Large-scale high-numerical-aperture super-oscillatory lens fabricated by direct laser writing lithography. RSC Adv. 2018, 8, 20117-20123. [CrossRef]

32. Bohling, M.; Seiler, T.; Wdowiak, B.; Jahns, J.; Mohr, J.; Börner, M. Highly precise micro-retroreflector array fabricated by the LIGA process and its application as tapped delay line filter. Appl. Opt. 2012, 51, 5989-5995. [CrossRef] [PubMed]

33. Helfert, S.F.; Edelmann, A.; Jahns, J. Hollow waveguides as polarization converting elements: A theoretical study. J. Eur. Opt. Soc. 2015, 10, 15006. [CrossRef]

34. Helfert, S.F.; Seiler, T.; Jahns, J.; Becker, J.; Jakobs, P.; Bacher, A. Numerical simulation of hollow waveguide arrays as polarization converting elements and experimental verification. Opt. Quantum Electron. 2017, 49, 313. [CrossRef]

35. Belle, S.; Helfert, S.F.; Hellmann, R.; Jahns, J. Hollow waveguide array with subwavelength dimensions as a space-variant polarization converter. J. Opt. Soc. Am. B 2019, 36, D119-D125. [CrossRef]

36. Khan, M.Z.H.; Nakanishi, T.; Osaka, T. Effects of chemical treatment of indium tin oxide electrode on its surface roughness and work function. Surf. Coat. Technol. 2014, 244, 189-193. [CrossRef]

37. Kang, J.W.; Lee, S.P.; Kim, D.G.; Lee, S.; Lee, G.H.; Kim, J.K.; Park, S.Y.; Kim, J.H.; Kim, H.K.; Jeong, Y.S. Reduction of Series Resistance in Organic Photovoltaic Using Low Sheet Resistance of ITO Electrode. ECS Solid State Lett. 2009, 12, H64. [CrossRef]

38. Wang, D.H.; Kyaw, A.K.K.; Gupta, V.; Bazan, G.C.; Heeger, A.J. Enhanced Efficiency Parameters of SolutionProcessable Small-Molecule Solar Cells Depending on ITO Sheet Resistance. Adv. Energy Mater. 2013, 3, 1161-1165. [CrossRef]

39. Choi, M.; Jo, K.; Yang, H. Effect of Different Pretreatments on Indium-Tin Oxide Electrodes. Bull. Korean. Chem. Soc. 2013, 34, 421-425. [CrossRef]

40. Yu, H.Y.; Feng, X.D.; Grozea, D.; Lu, Z.H.; Sodhi, R.N.S.; Hor, A.M.; Aziz, H. Surface electronic structure of plasma-treated indium tin oxides. Appl. Phys. Lett. 2001, 78, 2595-2597. [CrossRef]

41. Han, D.M.; Lee, J.H.; Jeong, K.H.; Lee, J.G. Effects of substrate bias power on the surface of ITO electrodes during $\mathrm{O} 2 / \mathrm{CF} 4$ plasma treatment and the resulting performance of organic light-emitting diodes. Met. Mater. Int. 2010, 16, 627-632. [CrossRef]

Publisher's Note: MDPI stays neutral with regard to jurisdictional claims in published maps and institutional affiliations.

(C) 2020 by the authors. Licensee MDPI, Basel, Switzerland. This article is an open access article distributed under the terms and conditions of the Creative Commons Attribution (CC BY) license (http:/ / creativecommons.org/licenses/by/4.0/). 